\title{
Treatment of severe lipophilic intoxications with intravenous lipid emulsion: a case series (20II-20|4)
}

\author{
Michael D Becker \\ Brian C Young \\ Emergency and Critical Care, \\ Animal Specialty Group, \\ Los Angeles, CA, USA
}

This article was published in the following Dove Press journal:

Veterinary Medicine: Research and Reports

30 October 2017

Number of times this article has been viewed
Correspondence: Michael D Becker ACCESS Specialty Animal Hospital, 9599 Jefferson Boulevard, Los Angeles, CA 90232, USA

$\mathrm{Tel}+\mathrm{I} 3105586100$

Fax +l 3105586199

Email mike.bek@gmail.com
Abstract: The objective of this retrospective study was to describe the responses to treatment with intravenous lipid emulsion (ILE) and the outcomes for a variety of severe intoxications. This case series includes 10 client-owned animals, 9 dogs and 1 cat, that underwent treatment with ILE for a variety of severe intoxications over a 4-year period. History, physical examination findings, clinical signs, clinicopathological test results, treatment, response to treatment, and outcome were recorded. Eight of the 10 patients survived to discharge. The toxicities included in this case series were baclofen, ivermectin and spinosad plus milbemycin oxime, baclofen and tadalafil, carbamate, methamphetamine, dextroamphetamine sulfate, amlodipine, bromethalin, and organophosphate. The two patients who died were intoxicated with bromethalin and an organophosphate. Six of the 10 patients developed lipemia secondary to ILE administration, and there were no other known adverse effects. Overall, ILE was a safe therapeutic option. This case series provides clinical evidence of successful treatment with ILE as an antidote for previously unpublished toxicities (amlodipine, carbamate, methamphetamine, and dextroamphetamine sulfate), additional evidence of success in treating baclofen and ivermectin toxicosis, as well as unsuccessful treatment of bromethalin and organophosphate toxicities.

Keywords: intravenous lipid emulsion, toxicity, amlodipine

\section{Introduction}

ILE has been used in human and veterinary medicine to treat a variety of intoxications in recent years. The first report of successful treatment with ILE in the veterinary literature is a puppy with moxidectin toxicity, published in 2009. ${ }^{1}$ Since then, several case reports and case series have been published reporting successful outcomes treating dogs and cats with ILE for various other toxicities, including lidocaine, ${ }^{2}$ permethrins, ${ }^{3-5}$ baclofen, ${ }^{6}$ ivermectin, ${ }^{7-9}$ diltiazem, ${ }^{10}$ and ibuprofen. ${ }^{11}$ In addition, two research studies using canine models have shown success using ILE as part of a resuscitation protocol for bupivacaine ${ }^{12}$ and verapamil ${ }^{13}$ toxicoses.

ILE consists of a sterile $20 \%$ fat emulsion manufactured as a source of intravenous calories and essential fatty acids. It is made up of $20 \%$ soybean oil, as well as egg phospholipids, glycerin, and water. The soybean oil consists of predominantly unsaturated fatty acids: linoleic acid, oleic acid, palmitic acid, $\alpha$-linolenic acid, and stearic acid.

There are several potential theorized mechanisms of action of ILE that have been previously reviewed in the veterinary literature. ${ }^{14-16}$ Briefly, the potential mechanisms include 1) sequestering the toxic substance in a new lipid compartment within the intravascular space (known as a "lipid sink"); 2) improving mitochondrial function by 
providing a source of fatty acids for metabolism; 3 ) providing cardiomyocytes with energy substrate; and 4) improving cardiomyocyte function by increasing intracellular calcium. A recent research study provided visual demonstration of the lipid sink effect by using a dye surrogate in place of bupivacaine and lidocaine. ${ }^{17}$

Major adverse effects of ILE therapy are rare; however, there are numerous possible complications to consider. These include lipemia, pancreatitis secondary to lipemia, interference with lipid-soluble therapeutic drugs, immune cell dysfunction, development of ARDS or worsened oxygenation in ARDS patients, hypersensitivity reactions to soybean or egg protein, lipid emboli, thrombosis, thrombocytopenia, laboratory errors (false increases of glucose, methemoglobin, aspartate aminotransferase, amylase, phosphorus, or creatinine), fat overload syndrome (hyperlipidemia, hemolysis, jaundice, seizures, prolonged clotting time, thrombocytopenia, and fat embolism), sepsis secondary to bacterial growth in the bag or intravenous line, and phlebitis. ${ }^{14-16,18-20}$ Safety studies have shown that the LD50 of ILE in rats is $68 \mathrm{~mL} /$ $\mathrm{kg} .{ }^{21}$ The ASPCA Animal Poison Control-recommended dose for dogs is a $1.5 \mathrm{~mL} / \mathrm{kg}$ bolus followed by $0.25-0.5 \mathrm{~mL} / \mathrm{kg} /$ minute CRI, which has been extrapolated from experimental studies $^{12,13,21,22}$ and recommended human dosages. ${ }^{23}$ These guidelines are based on bupivacaine toxicosis studies in dogs and rats. ${ }^{12,21}$

Overall, multiple veterinary reviews have concluded that ILE therapy is safe and should be considered for severe intoxications. ${ }^{14-16}$ However, because ILE is a new therapy that has not been fully prospectively evaluated, it is recommended to be reserved for severe cases in which standard treatment fails.

The objective of this case series is to describe the use of ILE to treat dogs and cats with severe intoxications presenting to a busy private urban ED.

\section{Materials and methods}

Case selection

Hospital medical records were searched for any patient receiving ILE between January 1, 2011, and September 15,2014 . The search revealed 11 possible cases for inclusion. One case was excluded from the study because it was deemed by the attending neurologist that the patient's clinical signs were more likely due to primary intracranial disease rather than the initially suspected toxin ingestion. Confirmatory testing such as a magnetic resonance imaging of the brain or cerebral spinal fluid analysis was not performed. Ten patients (nine dogs and one cat) with 11 different lipophilic intoxications were included in the study (Table 1). The toxicities included baclofen (two cases), baclofen and tadalafil, ivermectin and spinosad plus milbemycin oxime, bromethalin, methomyl (a carbamate), disulfoton (an organophosphate), methamphetamine, dextroamphetamine sulfate (Adderall; Shire, St Helier, NJ, USA), and amlodipine.

\section{Review of medical records}

All 10 patients were selected by the attending clinician to receive ILE either due to severe clinical signs associated with toxicity or due to a potentially lethal dose of toxin. A data sheet for each patient was completed from what was available in the medical records. The data sheet consisted of signalment, prior medical history and medications, intoxication and dose when available, treatments received prior to referral, patient vitals, clinical signs, problem list, labwork performed, DBP before and after ILE treatment, MGCS, ILE dose received, time after ingestion before ILE was initiated, time after ILE administration before improvement of clinical signs, any observed complications of ILE treatment, and outcome. ${ }^{24}$

Table I Signalment, MGCS score, and outcome of patients ingesting various lipid-soluble toxins.

\begin{tabular}{|c|c|c|c|c|c|c|c|}
\hline $\begin{array}{l}\text { Case } \\
\text { number }\end{array}$ & $\begin{array}{l}\text { Age } \\
\text { (years) }\end{array}$ & Sex & Breed & Species & Toxin & $\begin{array}{l}\text { Modified } \\
\text { Glasgow Coma } \\
\text { Scale }(0-18)\end{array}$ & $\begin{array}{l}\text { Survival to } \\
\text { discharge? }\end{array}$ \\
\hline $\mathrm{I}$ & 1 & Intact male & Chihuahua & Canine & Baclofen & 16 & Yes \\
\hline 2 & 10 & Castrated male & Boston Terrier & Canine & Ivermectin, spinosad & 12 & Yes \\
\hline 3 & 2 & Spayed female & $\begin{array}{l}\text { American } \\
\text { Staffordshire Terrier }\end{array}$ & Canine & Baclofen, tadalafil & 8 & Yes \\
\hline 4 & 12 & Intact female & Maltese & Canine & Bromethalin & 8 & No \\
\hline 5 & 2 & Spayed female & English Bulldog & Canine & Carbamate & 10 & Yes \\
\hline 6 & 0.5 & Intact male & Shih Tzu & Canine & Methamphetamine & 17 & Yes \\
\hline 7 & 9 & Spayed female & Golden Retriever & Canine & Organophosphate & 16 & No \\
\hline 8 & 8 & Spayed female & Jack Russell Terrier & Canine & Amphetamine & 16 & Yes \\
\hline 9 & 0.33 & Intact male & Yorkshire Terrier & Canine & Amlodipine & 18 & Yes \\
\hline 10 & 0.29 & Intact female & Domestic Short-Hair & Feline & Baclofen & 12 & Yes \\
\hline
\end{tabular}

Abbreviation: MGCS, Modified Glasgow Coma Scale. 
The ILE protocol utilized by all patients in this study is as follows: a $500 \mathrm{~mL}$ or $1 \mathrm{~L}$ bag of $20 \%$ fat emulsion (Intralipid; Baxter Healthcare Corporation, Deerfield, IL, USA) was spiked and connected to the patient's intravenous catheter using aseptic technique. The ILE was run through a $1.2 \mu \mathrm{m}$ filter (Non-DEHP Extension Set with 1.2 Micron Downstream Filter; Baxter Healthcare Corporation) prior to reaching the patient. A dedicated line and intravenous catheter was used only for the ILE during infusion. The ILE bag was refrigerated after use for a maximum of 24 hours before being discarded. The dosage used varied slightly for most patients in the study and was based on recommendations of the ASPCA and previously published literature. Most patients received a $1.5 \mathrm{~mL} / \mathrm{kg}$ bolus followed by a CRI of $0.25 \mathrm{~mL} / \mathrm{kg} /$ minute for 30-60 minutes. Some patients had this protocol repeated once. One patient had a $1 \mathrm{~mL} / \mathrm{kg} /$ hour infusion continued for 12 hours after the initial dose. All variations in dosage were determined by the attending clinician based on the severity of the specific patient's clinical signs.

\section{Results \\ Case I}

A 1-year-old male intact Chihuahua presented for evaluation due to baclofen intoxication. The patient ingested $13.8 \mathrm{mg} / \mathrm{kg}$ (lethal dose in dogs $8-16 \mathrm{mg} / \mathrm{kg}$ ). Clinical signs included vomiting shortly after ingestion, ptyalism, severe ataxia, tremors, and stuporous mentation. He was treated with intravenous diazepam and crystalloids by the referring veterinarian prior to presentation. At the time of arrival, the patient was hypotensive (DBP $80 \mathrm{mmHg}$ ), hyperreactive, and minimally responsive. The MGCS was 16.

Due to the severity of neurologic signs, and the lethal amount ingested, the patient was treated with ILE. He received a $1.5 \mathrm{~mL} / \mathrm{kg}$ bolus followed by $0.25 \mathrm{~mL} / \mathrm{kg} /$ minute for 45 minutes of a $20 \%$ lipid emulsion. Other treatments included a $22 \mathrm{~mL} /$ $\mathrm{kg}$ bolus of crystalloids and $13.2 \mathrm{~mL} / \mathrm{kg}$ of activated charcoal through a nasogastric tube. DBP improved to $156 \mathrm{mmHg}$ within 1 hour of initial treatments. Ataxia, dull mentation, and tremors resolved within 3 hours of lipid infusion completion. The patient remained intermittently hyperreactive for 12 hours. The patient was discharged from the hospital 36 hours after arrival hemodynamically stable, with normal neurologic status. The patient developed lipemia that resolved within 8 hours and no other side effects of ILE administration.

\section{Case 2}

A 10-year-old male neutered Boston Terrier presented for ivermectin and spinosad plus milbemycin oxime toxicities. He had been previously diagnosed with demodicosis by the referring veterinarian and treated with ivermectin for 1 month. During the month-long treatment course, the patient developed episodes of mild ataxia, ptyalism, and mydriasis. He was tested for the ABCB1 gene mutation by the referring veterinarian and was found to be normal type, so the ivermectin was continued. His owner then applied an appropriate dose of a monthly topical flea product containing spinosad and milbemycin oxime (Trifexis; Elanco Animal Health, Eli Lilly and Company, Indianapolis, IN, USA). Shortly thereafter, he presented directly to the ED for muscle tremors, dull mentation, ptyalism, and blindness. The MGCS was 12 .

Shortly after arrival, the patient developed aspiration pneumonia secondary to vomiting. Throughout the first night of hospitalization, he became progressively obtunded but maintained normal ventilation. He remained hemodynamically stable and was treated for aspiration pneumonia with parenteral antimicrobials and oxygen. At 14 hours after presentation, the decision to treat with ILE was made due to worsening mental status as well as tremors that were nonresponsive to midazolam or methocarbamol. He received a $1.5 \mathrm{~mL} / \mathrm{kg}$ bolus followed by $0.25 \mathrm{mg} / \mathrm{kg} /$ minute for 90 minutes. Five hours after initiating ILE, the patient was responsive and able to stand and the tremors had resolved. He remained blind, but the dazzle reflex returned within 12 hours; he regained vision over 2-3 days. The patient developed lipemia that persisted for 3 days before clearing.

\section{Case 3}

A 2-year-old female spayed American Staffordshire Terrier mix presented as a transfer from a referring veterinarian 5 days after severe baclofen and tadalafil (Cialis; Lilly USA, LLC., Indianapolis, IN, USA) toxicities. The patient ingested a bottle of her owner's baclofen (total dose $56.2 \mathrm{mg} / \mathrm{kg}$ ) and an unknown amount of tadalafil (maximum suspected dose $7.5 \mathrm{mg} / \mathrm{kg}$ ). She initially presented to the referring emergency clinic vocalizing, laterally recumbent, and obtunded. The MGCS was 8. She was maintained on a surgical ventilator for 72 hours without sedation due to her comatose state. She was then removed from mechanical ventilator support and managed with flow-by oxygen for hypoxemia secondary to aspiration pneumonia. On the following day, she developed paddling and vocalizing that required sedation. She was reintubated and maintained on isoflurane inhalant initially, followed by a propofol CRI for 24 hours, prior to referral.

Upon arrival, DBP was $118 \mathrm{mmHg}$. An attempt was made to wean the patient from propofol sedation, but she began paddling and vocalizing. VBG analysis revealed hypoventilation $\left(\mathrm{PvCO}_{2} 66 \mathrm{mmHg}\right)$. At that time, the patient was reanesthetized and started on PPV. ILE was immediately 
instituted despite the length of time postintoxication (5 days). The patient received a $1.5 \mathrm{~mL} / \mathrm{kg}$ bolus followed by $0.25 \mathrm{~mL} /$ $\mathrm{kg} / \mathrm{minute}$ for 30 minutes, and then, the CRI was continued at a low rate of $1 \mathrm{~mL} / \mathrm{kg} /$ hour for 12 hours in addition to partial parenteral nutrition with a commercial product (Procalamine; B. Braun Medical Inc., Bethlehem, PA, USA). The patient was successfully weaned from PPV 12 hours after presentation because of return to normal ventilation. Signs of seizure-like activity such as paddling had resolved, and the lipids were discontinued. The patient remained hospitalized for 4 days. When first weaned from anesthesia, she was quiet but responsive, blind, and nonambulatory. Over the ensuing 3 days, she slowly recovered vision and ambulation with a mild proprioceptive deficit in the left pelvic limb. On recheck examinations, the patient returned to normal neurologic status over the next 2 weeks.

\section{Case 4}

A 12-year-old female intact Maltese was referred to the ED for bromethalin ingestion (Tomcat with Bromethalin; Motomco, Madison, WI, USA). The patient vomited green material in the night prior, became lethargic in the following morning, and continued vomiting. She was hospitalized at the referring veterinarian, where she received intravenous fluids (crystalloids $4 \mathrm{~mL} / \mathrm{kg} /$ hour and 6\% hydroxyethyl starch $1 \mathrm{~mL} / \mathrm{kg} /$ hour), famotidine $1.25 \mathrm{mg} / \mathrm{kg}$ intravenous (IV), maropitant $1.25 \mathrm{mg} / \mathrm{kg} \mathrm{IV}$, buprenorphine $0.015 \mathrm{mg} / \mathrm{kg} \mathrm{IV}$, and vitamin K1 $2.25 \mathrm{mg} / \mathrm{kg}$ subcutaneous. Six hours after presentation, she began vocalizing, her condition deteriorated, and she was transferred to the referral hospital.

On presentation, the patient was obtunded and laterally recumbent with delayed PLR OU, absent menace reflex OU, and anisocoria. The MGCS was 8. Rectal examination revealed green material in the stool consistent with the rodenticide. The patient was hypothermic $\left(21.7^{\circ} \mathrm{C}\right)$, but other vitals were stable.

The patient was treated with mannitol $1 \mathrm{~g} / \mathrm{kg}$ IV as well as a rectal enema to remove as much material from the gastrointestinal tract as possible. Approximately 2 hours after presentation, the patient received a $1.5 \mathrm{~mL} / \mathrm{kg}$ bolus of ILE, followed by a CRI of $0.25 \mathrm{~mL} / \mathrm{kg} /$ minute for 60 minutes. The same ILE protocol was repeated 7 hours after the first dose.

In the following morning, the PLR had improved and the anisocoria had resolved, but the patient remained mentally abnormal and occasionally vocalized inappropriately. The patient developed possible signs of the Cushing reflex (bradycardia, heart rate [HR] 40 beats/minute [bpm], and mild hypertension, DBP $154 \mathrm{mmHg}$ ). She received a dose of mannitol and was then intermittently agitated and tachycardic or obtunded and bradycardic. Signs of the Cushing reflex persisted (HR $60 \mathrm{bpm}$, DBP $160 \mathrm{mmHg}$ ), and later that afternoon when her owners visited, the patient remained mentally inappropriate and unresponsive. They elected humane euthanasia due to financial constraints and a poor prognosis.

\section{Case 5}

A 2-year-old female spayed English Bulldog presented immediately after ingesting a liquid fly repellant solution containing methomyl, a carbamate compound. The owner's child mistakenly poured the solution in the patient's water bowl. The patient consumed the product and immediately started seizuring and foaming at the mouth and collapsed. She initially presented to her referring veterinarian not breathing and was intubated for manual ventilation. She was administered diazepam and methocarbamol for tremors and began breathing on her own. The MGCS was 10. She was then transferred (still intubated) to the referral hospital.

On presentation, she was still tremoring and vomited several times. She was treated with a dose of midazolam and started on a methocarbamol CRI to control tremoring and also administered atropine to control parasympathetic nervous system signs. ${ }^{25}$ Approximately 4 hours after presentation, she was still tremoring despite methocarbamol, and due to an absent gag reflex, she remained intubated without sedation. At that time, ILE was administered. She received a $1.5 \mathrm{~mL} / \mathrm{kg}$ bolus followed by a CRI of $0.25 \mathrm{~mL} / \mathrm{kg} /$ minute for 60 minutes. At the end of the 60-minute infusion, the patient began chewing at the endotracheal tube and was extubated. The patient was alert, and no longer required methocarbamol. All clinical signs of toxicity dramatically improved. She remained hospitalized for 36 hours for the treatment of aspiration pneumonia and was discharged with no lingering clinical signs.

\section{Case 6}

A 6-month-old male intact Shih Tzu presented for ingestion of an unknown amount of methamphetamine. Another dog in the household died from methamphetamine toxicity prior to arrival. Upon presentation, he was disoriented, agitated, and circling to the right. The MGCS was 17 . Physical examination revealed hyperthermia $\left(27.9^{\circ} \mathrm{C}\right)$ and tachycardia $(180 \mathrm{bpm})$, and $\mathrm{VBG}$ analysis revealed metabolic acidosis $\left(\mathrm{HCO}_{3}{ }^{-}\right.$ $16.8 \mathrm{mmol} / \mathrm{L}$ ). A urine drug test (Multi Drug Screen Test; Easy At Home Medical, Burr Ridge, IL, USA) confirmed methamphetamine ingestion. He was initially treated with acepromazine $0.07 \mathrm{mg} / \mathrm{kg}$ IV for anxiety as well as a $22 \mathrm{~mL} /$ $\mathrm{kg}$ intravenous crystalloid bolus. The hyperthermia resolved, 
but he remained anxious and circling. Approximately 5-10 hours after suspected ingestion of methamphetamine, ILE was initiated. The patient received a $1.6 \mathrm{~mL} / \mathrm{kg}$ bolus followed by a $0.25 \mathrm{~mL} / \mathrm{kg} /$ minute infusion for 30 minutes. The mentation abnormalities (including anxiety and decreased responsiveness), as well as circling, resolved over 1-2 hours after ILE was administered. The patient remained hospitalized overnight and was discharged with normal neurologic status and hemodynamic status on the following day.

\section{Case 7}

A 9-year-old female spayed Golden Retriever with no previous medical history presented to the ED after suspicion for ingesting a rose fertilizer (2-in-1 Systemic Rose and Flower Care; Bayer Advanced, Research Triangle Park, NC, USA) containing disulfoton, an organophosphate, that the owner had put out in the garden that day. The owner saw the patient sniffing in the area where the fertilizer was spread. The first clinical sign noted several hours after the possible ingestion was profuse vomiting. At that time, the owner called the ASPCA Animal Poison Control and was advised to seek veterinary attention immediately. Upon arrival, the patient was diffusely tremoring and hyperthermic $\left(27.2^{\circ} \mathrm{C}\right)$. The patient had a delayed PLR OU but was responsive, and the rest of the neurologic examination was unremarkable. A 3/6 left holosystolic heart murmur was noted, with no other significant physical examination abnormalities. VBG analysis revealed hemoconcentration (packed cell volume $67 \%$ ), hyperlactatemia $(4.35 \mathrm{mmol} / \mathrm{L})$, and mild metabolic acidosis $\left(\mathrm{HCO}_{3}^{-} 17.6 \mathrm{~mol} / \mathrm{L}\right)$.

The patient was treated with methocarbamol $44 \mathrm{mg} / \mathrm{kg}$ IV for tremoring, a $22 \mathrm{~mL} / \mathrm{kg}$ crystalloid bolus, a dose of atropine $0.019 \mathrm{mg} / \mathrm{kg} \mathrm{IV}$, and active cooling with cool water. She responded well to initial treatments; her hyperthermia and tremoring resolved. She was admitted to the ICU for monitoring and received an ILE of $1.5 \mathrm{~mL} / \mathrm{kg}$ bolus followed by $0.25 \mathrm{~mL} / \mathrm{kg} / \mathrm{minute}$ for 30 minutes. Approximately 2 hours after being admitted, the patient received an additional dose of methocarbamol $44 \mathrm{mg} / \mathrm{kg}$ IV for facial tremors. One hour later, the patient had a seizure and received midazolam $0.5 \mathrm{mg} / \mathrm{kg}$ IV. The patient then went into cardiopulmonary arrest four times in 2 hours and was successfully resuscitated each time. At that point, her owner elected humane euthanasia due to a grave prognosis.

\section{Case 8}

A 4-year-old female spayed Jack Russell Terrier presented to the ED for anxiety, hyperexcitability, and moving her head from side to side after a walk. She was hyperthermic $\left(25.4^{\circ} \mathrm{C}\right)$, tachycardic $(160 \mathrm{bpm})$, and hypertensive (DBP $204 \mathrm{mmHg}$ ). On neurologic examination, she was extremely anxious and mentally inappropriate, pacing and head weaving (side to side), and mydriatic OU with slow PLRs. MGCS was 16. A urine drug screen tested positive for amphetamines, and the owner suspected she may have gotten into an old prescription bottle of dextroamphetamine sulfate used to treat attention deficit and hyperactivity disorder.

The patient was treated with acepromazine $0.02 \mathrm{mg} / \mathrm{kg}$ IV and a $20 \mathrm{~mL} / \mathrm{kg}$ crystalloid bolus IV on presentation. She received a second dose of acepromazine one hour later. Three hours after presentation, the clinical signs persisted, the patient was continuously circling in the cage and traumatizing herself by running into cage walls, so the decision was made to initiate ILE. She received a $1.5 \mathrm{~mL} /$ $\mathrm{kg}$ bolus over 5 minutes, followed by a CRI of $0.25 \mathrm{mg} /$ $\mathrm{kg} / \mathrm{minute}$ for 60 minutes. She received a second ILE infusion of the same dose 4 hours after the first ( 7 hours after presentation). The patient's hypertension improved within 3 hours after the first dose of ILE, but the tachycardia did not resolve until 7 hours after the second dose of ILE. The patient remained anxious throughout hospitalization; however, she stopped pacing and appeared progressively more mentally appropriate. The patient was discharged from the hospital, hemodynamically stable, approximately 22 hours after admission.

\section{Case 9}

A 4-month-old male intact Yorkshire Terrier presented to the ED 45 minutes after ingesting a $36.7 \mathrm{mg} / \mathrm{kg}$ dose of the owner's amlodipine. On presentation, the patient was severely tachycardic (260 bpm), hypotensive (DBP $50 \mathrm{mmHg})$, and hyperlactatemic $(6.63 \mathrm{mmol} / \mathrm{L})$. He received a $34 \mathrm{~mL} / \mathrm{kg}$ bolus of crystalloids IV followed by a $5 \mathrm{~mL} / \mathrm{kg}$ bolus of $6 \%$ hydroxyethyl starch. Recheck DBP after these boluses was unchanged at $50 \mathrm{mmHg}$. At that time, the ILE infusion protocol was administered. The patient received a $1.5 \mathrm{~mL} / \mathrm{kg}$ bolus over 5 minutes, followed by a CRI of $0.25 \mathrm{~mL} / \mathrm{kg} / \mathrm{minute}$ for 60 minutes. By the end of the ILE CRI, the DBP had stabilized at $96 \mathrm{mmHg}$. Four hours later, the lactate had improved to $2.08 \mathrm{mmol} / \mathrm{L}$. Over the next 12 hours, there were three episodes of mild hypotension (84-88 $\mathrm{mmHg}$ ) and the HR slowly improved to $115 \mathrm{bpm}$. Approximately 24 hours after presentation, the patient was hemodynamically stable and was discharged from the hospital. 


\section{Case 10}

A previously healthy 15 -week-old female intact domestic short-haired kitten presented to the ED for suspected baclofen toxicity. The clinical signs at home included depressed mentation, ataxia, vomiting, and urinating and defecating on herself. The owner found two partially dissolved baclofen pills. The kitten was initially presented to a referring veterinarian who administered crystalloids IV and contacted Pet Poison Hotline, before referring the patient. On physical examination, she was mildly hypotensive (DBP $80 \mathrm{mmHg}$ ) and hypothermic $\left(22^{\circ} \mathrm{C}\right)$. Neurologic examination revealed obtunded mentation, absent menace reflex OU, and absent PLR OU. Initially, a $22 \mathrm{~mL} / \mathrm{kg}$ crystalloid bolus was administered as well as naloxone $0.05 \mathrm{mg} / \mathrm{kg}$ IV. DBP improved to $120 \mathrm{mmHg}$; however, at recheck 1 hour later, the patient was again hypotensive (DBP $72 \mathrm{mmHg}$ ). At that time, ILE was administered. She received a $1.5 \mathrm{~mL} / \mathrm{kg}$ bolus followed by $0.25 \mathrm{~mL} / \mathrm{kg} /$ minute for 30 minutes. Six hours after the first ILE dose, there was no improvement in mental status, so she received a second dose of the same ILE protocol. Over 2 hours following the second ILE administration, a dramatic change in mental status was noted. The patient was able to rise and became alert and responsive. The cranial nerve abnormalities resolved. She was discharged from the hospital in the following morning, 22 hours after presentation.

\section{Discussion}

This case series describes 10 cases of different toxicities that showed varying responses to ILE treatment. In each case, the decision to treat with ILE was made either due to severe neurologic abnormalities, and hemodynamic instability or due to ingestion of a known lethal dose of toxin. Eight of the 10 patients survived to discharge. The two patients that died were intoxicated with bromethalin rodenticide and an organophosphate pesticide. The toxins that were most responsive to ILE were amlodipine, baclofen, carbamate, and ivermectin. To the authors' knowledge, these are the first reported veterinary cases using ILE to treat amlodipine, bromethalin, carbamate, organophosphate, and amphetamine toxicities.

Two of the 10 cases receiving ILE infusion died or were euthanized. The first of these cases (Case 4) was due to bromethalin rodenticide ingestion. Bromethalin's mechanism of action is to inhibit oxidative phosphorylation in the central nervous system (CNS), depleting adenosine triphosphate and resulting in cerebral edema. ${ }^{26}$ Once severe clinical signs develop, treatment is generally not effective. ${ }^{27}$ Case 4 appeared to have ingested bromethalin the day prior to referral because she had vomited some of the rodenticide the night prior to presentation. Bromethalin is highly lipid soluble with a long half-life in dogs of 5-6 days. ${ }^{26}$ In theory, Case 4 could have potentially responded to ILE by neutralizing residual bromethalin or bromethalin metabolites in the patient's CNS. This patient received two total doses of the standard ILE protocol, and some neurologic improvement did occur. It is also possible that Case 4 may have responded if more aggressive ILE therapy had been administered (eg, ILE dosages every 4 hours or ILE continuous rate infusion); however, this is merely speculative. Another possible outcome from Case 4 is that she may have responded to earlier ILE administration before more neuronal cell death occurred. It remains to be determined if bromethalin is a toxin that can be treated with ILE.

The second case that died in the hospital was due to organophosphate toxicity (Case 7). Organophosphates are used as antiparasitic medications or as pesticides. Clinical signs of toxicity are mainly due to their anticholinesterase activity in the blood and brain. ${ }^{28}$ 2-PAM (pralidoxime chloride) may have helped this patient, but the success of its use is controversial, depends on the timing of intoxication, and is not recommended by the ASPCA Animal Poison Control in this case. ${ }^{29,30}$ In theory, due to their high lipophilicity, organophosphates may be responsive to treatment with ILE. Similar to the patient in Case 4 who died of bromethalin toxicosis, this patient may have benefited from more aggressive ILE therapy.

The patient in Case 2 received multiple agents that may have contributed to his clinical signs. He was showing mild signs of ivermectin toxicity (mild ataxia, ptyalism, and mydriasis) during the first month of treatment with ivermectin. He then developed much more severe clinical signs (tremors, obtundation, and blindness) after being given a topical medication containing spinosad plus milbemycin oxime. This likely occurred due to a drug interaction between ivermectin and spinosad. Spinosad has been shown to cause delayed secretion of ivermectin from the body as well as from the CNS, via inhibition of P-glycoprotein efflux pumps. ${ }^{31}$ Milbemycin has similar potential neurologic side effects as ivermectin in dogs in overdose situations, however, is deemed safer than ivermectin in canine patients in general, as well as canine patients with the ABCB1- $\Delta$ genotype. As this patient received an appropriate dose of milbemycin, it appears unlikely to have contributed significantly to the clinical signs; however, it cannot be completely ruled out as contributing to the signs.

The timing of ILE administration after intoxication varied among the patients in this study. This is expected based on several variables for each case, including timing 
of patient evaluation by a veterinarian, timing of transfer to the referral hospital, and timing of the attending clinician's determination of when to start ILE. In some cases, ILE infusion was administered as soon as possible after admission; in others, it was not initially deemed necessary until the patient's status declined or the patient did not respond to initial therapy. While it is reasonable to assume that the sooner ILE is administered, the more effective it will be, this has not yet been shown in a controlled clinical trial. In addition, it is not known how long after intoxication ILE is still effective; this may vary depending on several factors such as toxin half-life, the patient's metabolism, concurrent medical conditions, and any additional medications that the patient is receiving. Of note, the patient in Case 3 received ILE infusion 5 days after severe baclofen intoxication and responded favorably. It was expected that the drug should have been completely metabolized by that time; however, the ILE infusion was administered to the patient due to the dire circumstances (need for PPV for hypoventilation) that were likely attributable to baclofen. Baclofen half-life in people is 2-4 hours but can increase to as long as 34 hours in overdose cases due to oversaturation of metabolic pathways. In addition, signs can continue after serum levels have normalized due to slow clearance from the CNS.$^{32}$ It is likely that due to the extremely high baclofen dosage ingested in Case 3, there was still residual drug affecting the patient's CNS. The tadalafil was unlikely to have contributed to the patient's neurologic status, as she had ingested a much lower dose compared to the baclofen.

In addition, Case 3 received a low-dose ILE CRI of $1 \mathrm{~mL} /$ $\mathrm{kg}$ /hour for 12 hours after the initial standard ILE administration. The CRI was continued for 12 hours by the attending clinician to provide parenteral nutrition to the patient (in addition to a commercial product containing only amino acids and carbohydrates) and potentially additional benefit in treating the baclofen intoxication. This extra amount of ILE was small in comparison to the standard rate of $0.25 \mathrm{~mL} /$ $\mathrm{kg} /$ minute; however, it may have benefited the patient. It is also possible that the low-dose extended CRI, as opposed to a bolus then 1 hour infusion, may have some as yet unknown benefit.

There are several theorized complications with ILE therapy, many of which have been determined from clinical trials of patients receiving parenteral nutrition. The only complication noted for any patient in this study was the development of lipemia, seen in six of the 10 patients. Any patient who developed lipemia was not administered subsequent ILE doses until clearance of lipemia, even if clinically indicated, due to concern for increased risk of complications. To the authors' knowledge, there has been no reported complication in veterinary patients other than lipemia secondary to ILE as a treatment for toxicities. Complications are rarely reported in the human literature and typically occur when ILE is administered at higher doses than those used in this study. Commonly reported complications in people include lipemia, hypertriglyceridemia, pancreatitis, and interference with laboratory analyses. ${ }^{33,34}$ Lipemia may be the simplest indicator; a patient is at risk of developing other more serious complications, but a correlation has not been shown. No such complication (other than lipemia) occurred in any patient in this study. Overall, ILE therapy was considered safe in all patients, in agreement with previous veterinary case reports and reviews.

The use of in-line filters is recommended for parenteral nutrition but has not yet become standard of care for ILE infusion. Current recommendations advise using a $1.2 \mu \mathrm{m}$ filter for lipid-containing infusions. ${ }^{35}$ There is some controversy regarding the use of in-line filters in human medicine. The proposed benefits include removing particulate matter, lipid globules, microbial contamination, and air from the infusion solution. Arguments against using in-line filters include increased cost and a potential additional source of contamination. A prospective randomized controlled trial has yet to be performed in humans, but research trials in animals have been done. Many different enteral solutions, including lipid emulsions, contain excessive amounts of particles that originate from manufacturing, packaging, and storage. Particles disperse to different areas of the body based on their diameter and can deposit in pulmonary, splenic, and hepatic capillary beds. ${ }^{36}$ Possible adverse reactions resulting from the infusion of particles in parenteral nutrition include vessel occlusion, inflammatory responses, antigenic stimulation, and neoplastic sequelae. ${ }^{37} \mathrm{~A}$ human review concluded that there is ample evidence to recommend the use of filters to all patients receiving any form of parenteral nutrition to prevent complications. ${ }^{38}$

This case series is a retrospective study and, thus, has inherent limitations. First, the data collected for the study came from medical records that may have been incomplete or inaccurate. Second, patients received slightly different doses of ILE, at different time points after intoxication. Additional treatments, such as resuscitation protocols, were also not standardized among the patients in the study. This makes interpretation of the patients' responses to ILE more difficult. Despite the clinical impression of positive response to ILE, some of the patients may have shown similar courses 
of improvement receiving standard treatment without ILE. A set of controls would have allowed comparison of ILE infusion versus standard treatment without ILE administration. However, a prospective randomly controlled clinical trial may be difficult to complete as it may not be ethical to withhold a potentially lifesaving therapy from a dying patient. Another set of data that may have helped determine a true clinical response to ILE is the comparison of toxin serum concentration before and after ILE administration. This was not performed in any case included in this retrospective series.

\section{Conclusion}

ILE is an overall safe therapy that should be considered for a variety of toxicities that cause neurologic or cardiovascular instability in veterinary medicine. There may be a better dosage protocol that is both safe and more effective. Prospective randomized controlled trials are required to determine more information about the safety of ILE in dogs and cats as well as ideal dosing regimens. Until these trials are completed, the use of ILE should be reserved for severe cases or cases not responding to traditional therapies.

\section{Abbreviations}

ARDS, acute respiratory distress syndrome; ASPCA, American Society for the Prevention of Cruelty to Animals; CRI, constant rate infusion; DBP, Doppler blood pressure; ED, emergency department; ILE, intravenous lipid emulsion; MGCS, Modified Glasgow Coma Scale; OU, oculi uterque, Latin for "both eyes"; 2-PAM, 2-pyridine aldoxime methochloride; PPV, positive pressure ventilation; PLR, pupillary light reflex; VBG, venous blood gas

\section{Disclosure}

The authors report no conflicts of interest in this work.

\section{References}

1. Crandell DE, Weinberg GL. Moxidectin toxicosis in a puppy successfully treated with intravenous lipids. J Vet Emerg Crit Care. 2009;19(2):181-186.

2. O’Brien TQ, Clark-Price SC, Evans EE, Di Fazio R, McMichael MA. Infusion of a lipid emulsion to treat lidocaine intoxication in a cat. $J$ Am Vet Med Assoc. 2010;237(12):1455-1458.

3. Haworth MD, Smart L. Use of intravenous lipid therapy in three cases of feline permethrin toxicosis. J Vet Emerg Crit Care. 2012;22(6): 697-702.

4. Kuo K, Odunayo A. Adjunctive therapy with intravenous lipid emulsion and methocarbamol for permethrins toxicity in 2 cats. J Vet Emerg Crit Care. 2013;23(4):436-441.

5. DeGroot WD. Intravenous lipid emulsion for treating permethrins toxicosis in a cat. Can Vet J. 2014;55(1):1253-1254.

6. Bates N, Chatterton J, Robbins C, et al. Lipid infusion in the management of poisoning: a report of 6 canine cases. Vet Rec. 2013;172(13):339.
7. Clarke DL, Lee JA, Murphy LA, Reineke EL. Use of intravenous lipid emulsion to treat ivermectin toxicosis in a Border Collie. J Am Vet Med Assoc. 2011;239(10):1328-1333.

8. Kidwell JH, Buckley GJ, Allen AE, Bandt C. Use of IV lipid emulsion for treatment of ivermectin toxicosis in a cat. J Am Anim Hosp Assoc. 2014;50(1):59-61.

9. Epstein SE, Hollingsworth SR. Ivermectin-induced blindness treated with intravenous lipid therapy in a dog. J Vet Emerg Crit Care. 2013;23(1):58-62.

10. Maton BL, Simmonds EE, Lee JA, Alwood AJ. The use of high-dose insulin therapy and intravenous lipid emulsion to treat severe, refractory diltiazem toxicosis in a dog. JVet Emerg Crit Care. 2013;12(3):321-327.

11. Bolfer L, McMichael M, Ngwenyama TR, O’Brien MA. Treatment of ibuprofen toxicosis in a dog with IV lipid emulsion. J Am Anim Hosp Assoc. 2014;50(2):136-140.

12. Weinberg G, Ripper R, Feinstein DL, Hoffman W. Lipid emulsion infusion rescues dogs from bupivacaine-induced cardiac toxicity. Reg Anesth Pain Med. 2003;28(3):198-202.

13. Bania TC, Chu J, Perez E, et al. Hemodynamic effects of intravenous fat emulsion in an animal model of severe verapamil toxicity resuscitated with atropine, calcium, and saline. Acad Emerg Med. 2007;14:105-111.

14. Fernandez AL, Lee JA, Rahilly L, Hovda L, Brutlag AG, Engebretsen $\mathrm{K}$. The use of intravenous lipid emulsion as an antidote in veterinary toxicology. J Vet Emerg Crit Care. 2011;21(4):309-320.

15. Gwaltney-Brant S, Meadows I. Use of intravenous lipid emulsions for treating certain poisoning cases in small animals. Vet Clin North Am Small Anim Pract. 2012;42(2):251-262.

16. Kaplan A, Whelan M. The use of IV lipid emulsion for lipophilic drug toxicities. J Am Anim Hosp Assoc. 2012;48(4):221-227.

17. Papadopoulou A, Willers JW, Samuels TL, Uncles DR. The use of dye surrogates to illustrate local anesthetic drug sequestration by lipid emulsion a visual demonstration of the lipid sink effect. Reg Anesth Pain Med. 2012;37(2):183-187.

18. Thrall MA, Weiser G, Allison R, Campbell TW, editors. Veterinary Hematology and Clinical Chemistry. 2nd ed. Ames, IA, USA: Wiley-Blackwell; 2012.

19. Levine M, Skolnik AB, Ruha AM. Complications following anecdotal use of intravenous lipid emulsion therapy. J Med Toxicol. 2014;10(1): $10-14$.

20. Martin C, Gonzalez H, Ruiz S, et al. Acute respiratory distress syndrome following verapamil overdose treated with intravenous lipid emulsion: a rare life-threatening complication. Ann Fr Anesth Reanim. 2014;33(6):e101-e102.

21. Hiller DB, Di Gregorio G, Kelly K, et al. Safety of high volume lipid emulsion infusion: a first approximation of LD50 in rats. Reg Anesth Pain Med. 2010;35(2):140-144.

22. Weinberg GL, VadeBoncouer T, Ramaraju GA, et al. Pretreatment or resuscitation with a lipid infusion shifts the dose response to bupivacaine-induced asystole in rats. Anesthesiology. 1998;88(4):1071-1075.

23. Cave G, Harvey M, Graudins A. Review article: Intravenous lipid emulsion as antidote: a summary of published human experience. Emerg Med Aust. 2011;23(2):123-141.

24. Platt SR, Radaelli ST, McDonnell JJ. The prognostic value of the modified Glasgow Coma Scale in head trauma in dogs. J Vet Intern Med. 2001;15(6):581-584.

25. Draper WE, Bolfer L, Cottam E, McMichael M, Schubert T. Methocarbamol CRI for symptomatic treatment of pyrethroid intoxication: a report of three cases. J Am Anim Hosp Assoc. 2013;49(5):325-328.

26. Murphy MJ. Rodenticides. Vet Clin North Am Small Anim Pract. 2002;32(2):469-484.

27. Coppock R. Special Report. Advisory: bromethalin rodenticide - no known antidote. Can Vet J. 2013;54(6):557-558.

28. The Merck Veterinary Manual. Organophosphate toxicity. Available from: http://www.merckvetmanual.com/toxicology/insecticide-andacaricide-organic-toxicity/organophosphates-toxicity. Accessed October 17, 2014. 
29. Eyer P. The role of oximes in the management of organophosphorus pesticide poisoning. Toxicol Rev. 2003;22(3):165-190.

30. Eddleston M, Szinicz L, Eyer P, et al. Oximes in acute organophosphorus pesticide poisoning: a systematic review of clinical trials. $Q J \mathrm{Med}$. 2002;95(5):275-283.

31. Dunn ST, Hedges L, Sampson KE, et al. Pharmacokinetic Interaction of the antiparasitic agents ivermectin and spinosad in dogs. Drug Metab Dispos. 2011;39(5):789-795.

32. Khorzad R, Lee JA, Whelan M, et al. Baclofen toxicosis in dogs and cats: 145 cases (2004-2010). J Am Vet Med Assoc. 2012;241(8): 1059-1064.

33. Bucklin MH, Gorodetsky RM, Wiegand TJ. Prolonged lipemia and pancreatitis due to extended infusion of lipid emulsion in bupropion overdose. Clin Toxicol (Phila). 2013;51(9):896-898.
34. Johnson-Arbor K, Salinger L, Luczycki S. Prolonged laboratory interference after administration of intravenous lipid emulsion therapy. $J$ Med Toxicol. 2015;11(2):223-226.

35. Chan DL, Freeman LM. Parenteral nutrition. In: DiBartola SP (editor), Fluid, Electrolyte, and Acid-Base Disorders in Small Animal Practice. 4th ed. Elsevier Saunders; 2012:605-622.

36. Bethune K, Allwood M, Grainger C, et al. Use of filters during the preparation and administration of parenteral nutrition: position paper and guidelines prepared by a British pharmaceutical nutrition group working party. Nutrition. 2001;17(5):403-408.

37. Turco SJ, Davis NM. Clinical significance of particulate matter: a review of the literature. Hosp Pharm. 1973;8:137-139.

38. Ball PA. Intravenous in-line: filtering the evidence. Curr Opin Clin Nutr Met Care. 2003;6(3):319-325.
Veterinary Medicine: Research and Reports

\section{Publish your work in this journal}

Veterinary Medicine: Research and Reports is an international, peer-reviewed, open access journal publishing original research, case reports, editorials, reviews and commentaries on all areas of veterinary medicine. The manuscript management system is completely online and includes a very quick and fair peer-review system

\section{Dovepress}

Visit http://www.dovepress.com/testimonials.php to read real quotes from published authors.

Submit your manuscript here: http://www.dovepress.com/veterinary-medicine-research-and-reports-journal 